\title{
Comparison of the effectiveness of cognitive- behavioral group therapy with cognitive hypnosis on reducing depression in students with premenstrual syndrome
}

\begin{abstract}
Introduction: Premenstrual syndrome (PMS) is defined as a set of physical and psychological symptoms that occur during the Luteal phase of the menstrual cycle. Among this complaint, depression is more common and aversive. Therefore, this study was designed to reduce depression with comparison of the effectiveness of group cognitive behavioral therapy and group cognitive hypnotism female students with premenstrual syndrome .

Methods: The research was done semi-experimental method on female students of Rasht city in 2017. Available sampling is used and 30woman with premenstrual syndrome chose and divided to 3 group into 2 Experimental group and 1 control group random. The first experimental group, received 8-week group cognitive behavioral therapy also, second group received, hypnosis cognitive therapy group the same for and the third group received no special treatment: 8 item Premenstrual syndrome inventory and the Beck's questionnaires depression used in Pre-test and Post- test. Data were analysis by covariance analysis and Tukey.
\end{abstract}

Results: the result of the statistical show both of them (therapy) was effective on reducing depression in women with PMS ( $<<0 / 001)$. Also, cognitive behavioral group therapy (CBGT) in compared with hypnotism therapy was not different in reduce depression in woman with PMS significantly.

Conclusion: The result of the research show psychological therapies effected for Premenstrual syndrome and as regards to less complaint, recommit to use this therapy .In addition with similar effectiveness CBGT and hypnotherapy, the using hypnotism to Substitute CBGT is recommit

Keywords: premenstrual syndrome, PMS, group cognitive behavioral therapy, cognitive group hypnotism, depression
Volume 7 Issue 6 - 2017

Leila Moghtader

Department of Psychology, Islamic Azad University, Iran

Correspondence: Leila Moghtader, Assistant of professor, Department of Psychology, Rasht Branch, Islamic Azad University, Iran, Email moghtaderleila@yahoo.com

Received: December 06, 2017 | Published: December 27, 2017

\section{Introduction}

Most women experience some physical and psychological changes from a few days to two weeks before the onset of a menstrual syndrome, which is called premenstrual syndrome. This syndrome involves a series of symptoms including depression, panic, change in sleep pattern, memory disorders, irritability and early suffering, anxiety, weight gain, appetite changes, etc. ${ }^{1}$ These symptoms can be seen in women of any age and race. According to global statistics, nearly $85 \%$ of women experience at least one of the symptoms of premenstrual syndrome, which causes a clear decline in performance and a decline in the quality of life in the individual, family and society. ${ }^{2}$ This syndrome is a set of physical and psychosomatic symptoms that occurs during the luteal phase of the menstrual cycle and improves with the onset of menstruation or the first few days. ${ }^{3}$ In addition, it is one of the most common painful phenomena in women's reproductive years. ${ }^{4}$ The physical signs and symptoms of the syndrome are sufficiently intense to interfere with some aspects of life. ${ }^{5}$ Symptoms should be observed at least 10 days immediately prior to menstruation, and no interval between menstruation and ovulation should begin at least one week. ${ }^{6}$ More than $90 \%$ of women of reproductive age report mild premenstrual symptoms and at least one of these symptoms is repeated each month. Of these, 2 to 10 percent of the severity of their symptoms is to an extent that affects family status, social activity, and job opportunities. ${ }^{?}$

The proper recognition of this disorder and awareness of its various biological, psychological and social dimensions is the first step in overcoming the disadvantaged disadvantages of it. Most women tend to be more sensitive to their needs during premenstrual periods. In these situations, they can spend more time in expressing emotions and feelings, calming themselves and giving their priority to themselves and their needs. ${ }^{8}$ Women with premenstrual syndrome spend days each month with defects in their individual, marital, and work-life environments. Research has shown that these monthly symptoms lead to significant damage to employers and health insurance companies annually. ${ }^{9}$ The issue of non-medical treatment is not clear. In a study in Spain, the attitudes of 2018 women aged 15-49years to the problem of premenstrual problems and the way in which medical centers were treated by them was examined. Among these, $73.7 \%$ reported some of the mild symptoms of premenstrual syndrome, $8.9 \%$ had severe symptoms, and only $1.1 \%$ of them were seeking medical treatment. Research showed that $90.6 \%$ of women referred to medical centers were told that these symptoms are normal, They did not receive 
$20 \%$ of the treatment because the physician believed that these symptoms would be eliminated on their own and $12 \%$ received only general recommendations. The result is that women who suffer from premenstrual syndrome and premenstrual disorder usually do not seek medical treatment, and the number of people who do not receive a good response in many cases. ${ }^{10}$ Recent research has shown the role of non-pharmacological treatments in improving quality of life and reducing the symptoms of affected patients. Almost all nonpharmacological methods of meditation to yoga are rooted in ancient Asian culture. Their only exception is the regular muscle relaxation method developed by American physician Edmond Jacobson in 1934. ${ }^{11}$ Blake and Sallykvski Vogat (Blake, Salkovskis, Gath) described the use of cognitive-behavioral therapy for PMS treatment. They believe that women with PMS may interpret psychological events in a negative way. ${ }^{12}$ The use of cognitive-behavioral therapy in treating this syndrome makes women more likely to find compatibility with premenstrual changes. ${ }^{13}$ Among the psychological therapies of hypnosis therapy, especially in the field of women's disease, hypnosis can be used in the following problems: childbirth delivery, early birth control, abortion and curettage, vomiting control of pregnancy, control of bleeding, menstrual disorders, premenstrual syndrome, fetal pregnancy, Types of sexual deviations, fear of sexual intercourse in women, delays in ejaculation, sexual hatred and hunger. ${ }^{14}$

Hypnosis is a condition that results from attention, acceptance, and concentration, in which there must be degrees of three facets of the same time: 1) decomposition or abstraction, 2) absorption, 3) inclusiveness. $^{15}$ Cognitive Behavioral Hypnotherapy is the combination of hypnotherapy with cognitive-behavioral therapy. ${ }^{16}$ Cognitive Behavioral Hypnotherapy is based on the assumption that most psychological disturbances are a negative form of self-hypnosis, so that negative thoughts are accepted critically and even without informed knowledge. Cognitive-behavioral hypnotherapy involves several basic methods including relaxation, guided imagery, cognitive reconstruction, gradual desensitization, and the training of hypnosis skills. ${ }^{17}$ In a study conducted by Allsworth and his colleagues at the University of Washington on 446 women under the age of 45years, it was found that as a result of an increase in stressful events in life, menstrual irregularities and symptoms of premenstrual syndrome increase. ${ }^{18}$ In a study, researchers investigated the relationship between premenstrual syndrome, experienced stress levels, quality of life, and continued exercise. The results showed that women with severe symptoms of premenstrual syndrome have significantly higher stress and lower quality of life than women with a degree of mild symptoms of premenstrual syndrome. ${ }^{19}$ Considering the necessity of cognitive and behavioral changes in decreasing stress and depression and considering that menstruation is one of the periods of increasing tension and depression in women, this research is based on the use of non-pharmacological methods and using cognitive therapies Behavioral and hypnosis will contribute to reducing this incidence in women's monthly cycle of fertility.

\section{Methods}

This semi-experimental study was carried out on 30 women of reproductive age at the health center affiliated to Center No. 12 in Rasht in the second half of 2017. The study population consisted of all students of Azad University of Rasht in 2017 who completed the questionnaire of premenstrual syndrome in two successive cycles and had diagnostic criteria for this syndrome. Then 30 of the women with the highest depression score after completing the Beck Depression
Inventory were selected and randomly divided into 3 groups of two experimental and one control group. Then, for the first group, 8 sessions of cognitive-behavioral group therapy were conducted to reduce depression during the month. The second group received 8 sessions of cognitive hypnosis group therapy to reduce the monthly depression. The third group remained in control group waiting for treatment. The Beck Depression Inventory was then returned to each of the three groups. Finally, the data were analyzed using descriptive and inferential statistical tests (mean ANCOVA tests) and SPSS version 21 software. The instruments used in this study included a questionnaire for measuring the symptoms of premenstrual syndrome and Beck's depression and anxiety inventory. Inclusion criteria included the age of fertility, high cycle education and the absence of mental illness, and the exit criteria did not have a negative SPI for hypnosis testing. The following tests and tools were used to measure and measure the variables: 1-A researcher-made questionnaire for measuring pre-menstrual syndrome, 2- Beck Depression Inventory.

Pre-menstrual test questionnaire: This questionnaire uses an unconfirmed form of prospective registration. The importance and severity of menstrual symptoms derived from the Kistner ${ }^{20}$ Women's Disease Guide, which is approved by the use of the comments of 6 faculty members. Was prepared and completed in two successive cycles by the students. Beck Depression Inventory (BDI): Beck Depression Inventory (BDI) is one of the most commonly used instruments for measuring mental disorders. This questionnaire is a multidimensional tool, which has been compiled by 21 items in card and computer forms. This was first developed by Beck in 1961. The validity and reliability of this questionnaire were reviewed and documented in the years 1971, 1979, 1985, 1986. The validity of simultaneously with clinical grading for psychiatric patients indicates correlation coefficients between mean to high and $r=72 .{ }^{21}$ The correlation of this test with the Hamilton scale for depression (0.73), Zong's depression scale (0.76), and Minnesota Multiphasic Personally Inventory (MMPI) (0.42) were obtained. ${ }^{22}$ The score for this test is Likert, and each item has 4 options that are scored on a scale of 0 to 3 , and will vary the degree of depression from mild to severe. The maximum score in this questionnaire is 63 and at least zero. After scoring the questionnaires by the three groups of participants in the pretest and posttest stages, the results were analyzed using ANCOVA statistical methods using SPSS software. The results were analyzed.

\section{Results}

The data obtained from the demographic questionnaire showed the majority of tests over the age of 30, married and graduate students (Table 1). The results showed that cognitive-behavioral therapy is effective on any two-dimensional depression (Table 2). In addition, hypnotherapy is effective in reducing depression in the syndrome (Table 3). The results also showed that there is no significant difference between cognitive-behavioral therapy and hypnotherapy in reducing depression, which means that hypnosis therapy is as effective as cognitive-behavioral therapy in decreasing depression of premenstrual syndrome (Table 4). Table 1, in addition to the frequency of the variables "age", "education" and "marriage" in this study, in total represents three groups of 10 people, including two experimental groups and a control group, a total of 9 individuals Coarse 30years and 21 people over $30 y e a r s$ old. In addition, 7 of them were single and 21 were married. In the education variable, 5 had undergraduate degrees, 17 had a bachelor's degree, and 8 were postdoctoral. According to the results of the tables, there was a significant difference in the 
comparison of the mean of the three methods of treatment with depression using Ancova test and the value of $\mathrm{P}$ obtained in Table 4.

But in comparing binary with post hoc test, the first method (cognitive-behavioral therapy) with control group has a significant effect on depression $(\mathrm{P}=0.005)$. Compared with the control group $(\mathrm{p}=0.018)$, depression in the second group (hypnotherapy) was significantly different. In general, as shown in the table of analysis of variance, the analysis of variance shows that the effect of cognitive therapy and hypnotherapy on depression is significant. However, these two treatments have not significantly decreased depression.

Table I Comparison of the frequency of age, education and marital status in two experimental and control groups

\begin{tabular}{lllll}
\hline & Control Group & \multicolumn{2}{l}{ Second experimental group } & \multirow{2}{*}{$\begin{array}{l}\text { First test } \\
\text { group }\end{array}$} \\
\cline { 3 - 4 } & \multicolumn{2}{c}{ Frequency } & \\
\hline 4 & 2 & 3 & $<30$ & Age \\
6 & 8 & 7 & $>30$ & \\
3 & 3 & 1 & Single & Marital status \\
7 & 7 & 9 & Married & education \\
2 & 1 & 2 & Bachelor & \\
5 & 6 & 6 & MA & Total \\
3 & 3 & 2 & P.H.D & \\
10 & 10 & 10 & &
\end{tabular}

Table 2 Comparison of mean and standard deviation of the main variables of the research in the three groups in the pre-test and post-test stages

\begin{tabular}{|c|c|c|c|c|c|c|}
\hline \multicolumn{2}{|c|}{ Control Group } & \multicolumn{2}{|c|}{ Second Experimental group (Hypnotherapy) } & \multicolumn{3}{|c|}{ First test group (Cbt) } \\
\hline \multirow[t]{2}{*}{ Post-test } & Pre-exam & Post-test & Pre-exam & Post-test & Pre-exam & \\
\hline & & \multicolumn{4}{|c|}{ Mean \pm Standard Deviation } & Variable \\
\hline $42.50 \pm 2.84$ & $44.70 \pm 84.2$ & $45 \pm 8.33$ & $46 \pm 9 / 85$ & $10.15 \pm 6.26$ & $10.96 \pm 6.45$ & Depression \\
\hline
\end{tabular}

Table 3 Covariance analysis in determining and comparing the effect of cognitive behavioral therapy and depression hypnotherapy

\begin{tabular}{lllllll}
\multicolumn{2}{l}{ Control Group } & \multicolumn{4}{l}{ Second experimental group (Hypnotherapy) } & \multicolumn{4}{l}{ First test group (Cbt) } \\
\hline Post-test & Pre-exam & Post-test & Pre-exam & Post-test & Pre-exam & \\
& & Mean \pm Standard deviation & & & Variable \\
$42.50 \pm 2.84$ & $44.70 \pm 2.84$ & $45 \pm 8.33$ & $46 \pm 9 / 85$ & $10.15 \pm 6.26$ & $10.96 \pm 6.45$ & Depression \\
\hline
\end{tabular}

Table 4 Covariance analysis in determining and comparing the effect of cognitive behavioral therapy and depression hypnotherapy

\begin{tabular}{lllll}
\hline F & Average squares & Degrees of freedom & Sum of squares \\
\hline & Mean Square & DF & SS & \\
6.389 & 515.42 & 2 & 1030.38 & Depression \\
--- & 80.676 & 54 & 4356.5 & Error \\
\hline
\end{tabular}

\section{Discussion and conclusion}

Cognitive-behavioral therapy and group hypnosis were found to be effective in decreasing depression, and these two treatments did not show a significant difference in depression among women with premenstrual syndrome. This conclusion was similar to the results of Biggs research, Cognitive therapy has been found to focus on the emotions and behaviors that affect the treatment of this syndrome..$^{23}$ In explaining this emmi, it is possible to refer to the Contor research to investigate brain function in the premenstrual syndrome and mood disorder in the American Neuroscience Clinic. The comparison between women with dementia, women without dementia and women with severe premenstrual symptoms showed that mood disorders in the second half of the menstrual cycle were much more than the first half, and those with symptoms of syndrome. Before menstruation, they showed changes in attention, concentration and mood. The cerebrospinal fluid of these people showed that these changes are due to poor functioning of the frontal lobe and temporal lobe of the brain. Therefore, the similarity of the biological mechanisms that causes mood disorders with PMS-induced depression causes the effect of cognitive-behavioral therapy on both diagnosis. Cognitivebehavioral strategies affect cognitive behavior and stimulate the body to be vibrant and vibrant and activate the immune system in the body. Also, the effect of these strategies on mental health is related to cognitive processes, such as the kind of looking at life and the viewing of oneself. On the other hand, women's awareness about the symptoms of premenstrual syndrome and the physiologic process of menstruation can be increased. They will better adapt to 
the phenomenon of menstruation and physiological changes and change their attitude towards gender and related issues. On the other hand, one of the benefits of group training is the increase of social relationships. And, having participated in cognitive-behavioral group sessions, people have somewhat experienced the positive outcomes of social relationships, and by participating in meetings and using the views and solutions of others, they have broader views than There are issues raised in the group..$^{24}$ This was in line with researcher research that showed that the unconventional response to stressful situations in women with PMS is related to severe or chronic stressful events in their lives. In another study, researchers investigated the relationship between premenstrual syndrome, experienced stress, quality of life, and continued exercise. The results showed that women with severe symptoms of premenstrual syndrome were significantly more stressed and lower quality of life than women with mild symptoms of premenstrual syndrome. ${ }^{25}$ Halbreich in a study found similarities and relationships between the syndrome and mood disorders such as depression, panic disorder, major depression, and seasonal depression. A history of anxiety and mood disorders has been reported in half of these women, and the prevalence of depression in these women is higher than that of the normal population, which is consistent with the findings of the study. ${ }^{26}$ In addition, these findings are in line with the results of Panay's research, which stresses interpersonal relationships that increase the symptoms of this syndrome. ${ }^{8}$ In addition, with Allsworth's research, the effect of increasing the stressful events of life in menstrual irregularities and symptoms of premenstrual syndrome ${ }^{20}$ is consistent. In explaining the results it can be argued that the personality characteristics of women make them more susceptible to short-term changes in order to obtain satisfactory results and solve their problems. Additionally, knowledge of cognitive changes during the monthly periods and getting information about their ability to be controlled by the individual is one of the important reasons for the reduction of anxiety symptoms in these women.

Also, the findings of this study are in line with Kirkby's research findings, suggesting that teaching cognitive-behavioral coping skills can reduce the negative effects of symptoms of premenstrual syndrome and reduce these symptoms during the time period to maintain. ${ }^{27}$ This finding is in line with the results of Shahidi's research ${ }^{28}$ and in contradiction to the results of the research findings of Badleh et al., ${ }^{29}$ as they found that hypnotherapy has a therapeutic effect more than twice as much as conventional psychotherapy. However, many scholars and clinical experts have already observed that hypnosis creates a synergistic effect, especially when combined with cognitivebehavioral therapy. Hypnotherapy-based therapies provide dual power in cognitive-behavioral therapies. Suspension of critical thinking in hypnosis suggests the patient more inclined to accept persuasive conversations of behavioral cognitive therapy. Group cognitive behavioral hepno-therapy is generally similar to cognitive-behavioral therapy and benefits from its principles. In addition, the results of Morse and colleagues' research on the effectiveness of cognitivebehavioral therapy and drug therapy in premenstrual syndrome showed that coping skills training compared to mental and tone therapy in women with premenstrual syndrome More has been. Rapid early responses to drug therapy have been slowly reduced after 2 months, but the positive benefits of cognitive therapy have been significant after three months, which contradict the results of current research. ${ }^{30}$ In addition, with Helmi and colleagues' research, the aim of this study was to compare the effectiveness of cognitive-behavioral cognitivebehavioral therapy and cognitive-behavioral heiponotherapy in reducing the anxiety of Iranian children, which concluded that cognitive-behavioral hypnotherapy led to more anxiety reduction than cognitive behavioral therapy. This difference was significant. ${ }^{31}$ One of the research constraints that the researcher controlled was the interruption of hours of training with a crowd of clients at the health center, which causes distraction subjects. Specifically, therapeutic sessions with cognitive hypnosis, which require a relaxed atmosphere and focus, are sometimes held in unfavorable conditions. In addition, the low volume of test subjects and the inability to follow subjects over longer than the research limitations. Out of the constraints outside the control of the researcher, there was no similar research that made it difficult to compare, especially in the hypnotized subject group. The use of non-pharmacological treatments with regard to less complications and the presence of drug dependence and less relapse in eliminating physical and psychological symptoms in premenstrual syndrome is recommended. It is also recommended that women's awareness be promoted through the distribution of leaflets, pamphlets and training materials on the syndrome by health centers, welfare organizations and relevant counseling centers. Comparing other psychological treatments such as metacognitive therapy and schema therapy, and also comparing psychological treatments with drug therapies, is recommended in future research. Research in other cases of women's diseases such as menstruation and infertility in women, as well as infertility is suggested.

\section{Acknowledgements}

Thanks and appreciation: I am grateful to all the students participating in the research in perfection.

\section{Conflict of interest}

The author declares no conflict of interest.

\section{References}

1. Sadock BJ, Sadock VA. Kaplan and Sadock's Synopsis of Psychiatry. 7th ed. USA: Lippincott Williams and Wilkins; 2007. p.164-166.

2. Steinner M, Macdougall M, Brown E. The premenstrual symptoms screening tool (PSST) for clinicians. Arc Womens Ment Health 2003;6(3):203-209.

3. Thu M, Dias EO, Sawh S. Premenstrual syndrome among famale university students in thiland. $A U J T$. 2006;9(3):158-162.

4. Doglase S. premenstrual syndrome. Evidence based treatmet in family practice. Can Fam physician. 2002;48:1789-1797.

5. Dickerson lori M, Pamela JM, Melissa H Hunter. Premenstural syndrome. American Academy of Family physician. 2003;67(8):1743-1752.

6. Sadock BJ, Sadock GA. Comprehnsiue textbook of psychiatry. 8th ed philadelphia: USA: Lippincott williams and wilkinsi; 2005.

7. Nyberg S. How to Determine symptom severity in premenstrual syndrome: a combination of dalily ratings and interviews. Journal of Sexual Productive Healthcare. 2011;2(4):161-168.

8. Panay, Nick. Managing premenstrual syndrome. Womens Health Medicine. 2005;2(3):1-4

9. Dean B, Bornestein JE. A prospective assesment investigating the relationship between work and impairment with premenstrual syndrome. $J$ Occup Environ Med. 2005;46(7):649-656.

10. Lete I, Duenas J, Serrano I. Attitudes of Spanish Women toward premenstrual syndrome-premenstrual dysphoric disorder. European Journal of Obstetrics and Gynecology and Reproductive Biology. 2011;159(1):115118.

11. Seaward, Brian, Luke. Essentials of managing stress. 2nd ed. USA: Jones \& Barlett publisher; 2007. p. 22-28. 
12. Blake F, Salkovskis P, Gath D, et al. Cognotive therapy for premenstrual syndrome. J Psychosom Res. 1998;45(4):307-318.

13. Connolly M. Premenstrual syndrome: on update on definitions, diagnosis and management. Advances in psychiatric treatment. 2001;7(6):469477.

14. Hammond C. Hypnotic Inductions Suggestions. In: Alavi Fazel SK. 1st ed. Ahvaz: Tarava; 1991. p. 211-217.

15. Spiegel H, Greenleaf M. Personality style and hypnotizability: The fix flex continuum. Psychiatric Medicine. 1993;10(1):13-24.

16. Rabertson D. A brief introduction of cognitive-behavioral hypnotherapy; 2008.

17. Dowd T. Cognitive hypnotherapy. In: Fathi M editor. Mashhad. 1st ed. Bamshad, USA: john Wiley and sons; 2008. p. 120-128.

18. Allsworth JE. The influence of stress on menstrual cycle among newly. $J$ Adolescent Health. 2007;1175-1182.

19. Lustyk M, Widman L, Paschane A, et al. Stress, quality of life and physical activity in women with varying degrees of premenstrual syndrome. Women \& Health. 2004;39(3):35-44.

20. Ryan KJ Berkowitz R, S Barbieri RL. Kistner's Gynecology. In: Ghazijahani B, 1st ed. Esharat. Tehran; 1995. 442 p.

21. Abolghasemi A, Narimani M. Psychological tests. 11th ed. Ardabil: Bagh Rezvan; 2004. p. 265-266.

22. Beck AT, Epstein N, Brown G, et al. An inventory- for measuring clinical anxiety: Psychometric properties. J Consult Clini Psychol. 1988;56(6):893-897.
23. Biggs WS, Demut RH. Premenstural syndrome and premenstrual dyphoric disoder. Am Fam Physician. 2011;84(8):918-924.

24. Contor DS. Premenstrual syndrome: brain functional correlates to dysphoria in PMS. Clinical Neuro Psychology. 2000;15(8):699-700.

25. Lustyk M, Widman L, Paschane A, et al. Stress, quality of life and physical activity in women with varying degrees of premenstrual syndrome. Women \& Health. 2004;39(3):35-44.

26. Halbreich U, Borenstein J, Pearlstein T, et al. The prevalence, impairment, impact, and burden of premenstrual dysphoric disorder (PMS/ PMDD). Psychoneuroendocrinology. 2003;3:1-23.

27. Kirkby RJ. Changes in premenstrual symptoms and irrational thinking following cognitive behavioral coping skills training. J of consulting and clinical psychology. 1994;62(5):1026-1032.

28. Shahidi E. Hypnotherapy in premenstrual syndrome. $J$ Welfare and successfully. 2008;6(66):68-69.

29. Badeleh M, Fathi M, Aghamohaadian H, et al. Compare the effectiveness of cognitive behavioral therapy and Hypnotherapy to increase selfesteem in adulsness. J Fundamentals of Mental Health. 2012;15(3):412422.

30. Morse CA, Dennersttein L, Farrell E, et al. Comperhentohn of hormone therapy, coping skills training and relaxation for the relif of premenstrual syndrome. J Behav med. 1991;14(5):469-489.

31. Helmi K, Dehkordi M, Taghizadeh ME. Comparison of effectiveness of two methods of modular cognitive behavioral therapy and cognitive behavioral hypnotherapy in reducing Iranian children anxiety. Proceeding of the $1^{\text {st }}$ International Congress Hypnotism and Related Sciences, Iran; 2011 . 